Please cite this article as: Tourte, M. et al. (2021). Acid Hydrolysis for the Extraction of Archaeal Core Lipids and HPLC-MS Analysis. Bio-protocol 11(16): e4118. DOI: 10.21769/BioProtoc. 4118 .

\title{
Acid Hydrolysis for the Extraction of Archaeal Core Lipids and HPLC-MS Analysis
}

Maxime Tourte ${ }^{1}$, Philippe Schaeffer ${ }^{2}$, Vincent Grossi ${ }^{3}$ and Philippe M. Oger ${ }^{1, *}$

\author{
${ }^{1}$ Univ Lyon, INSA Lyon, CNRS, UMR 5240, F-69621, Villeurbanne, France; ${ }^{2}$ Univ. Strasbourg, CNRS, \\ UMR 7177, F-67000 Strasbourg, France; ${ }^{3}$ Univ Lyon, Univ. Lyon 1, ENSL, CNRS, UMR 5276 LGL-TPE, \\ F-69622 Villeurbanne, France \\ *For correspondence: philippe.oger@insa-lyon.fr
}

\begin{abstract}
[Abstract] Lipid membranes are essential cellular elements as they provide cellular integrity and selective permeability under a broad range of environmental settings upon cell growth. In particular, Archaea are commonly recognized for their tolerance to extreme conditions, which is now widely accepted to stem from the unique structure of their lipids. While enhancing the stability of the archaeal cell membrane, the exceptional properties of archaeal lipids also hinder their extraction using regular procedures initially developed for bacterial and eukaryotic lipids. The protocol described here circumvents these issues by directly hydrolyzing the polar head group(s) of archaeal lipids and extracting the resulting core lipids. Although leading to a loss of information on the nature of polar heads, this procedure allows the quantitative extraction of core lipids for most types of archaeal cells in an efficient, reproducible, and rapid manner.
\end{abstract}

Keywords: Archaea, Cell membrane, Core lipids, Acid hydrolysis, HPLC-MS, Extremophiles

[Background] Although found in all biotopes on Earth, Archaea are known as the main inhabitants of the most extreme environments, be it due to temperature, $\mathrm{pH}$, salinity, hydrostatic pressure, or other environmental stressors (Komatsu and Chong, 1998; Baba et al., 1999). This ability to cope with multiple extreme conditions has been associated with one of their most diagnostic features, i.e., the unique structure of their membrane lipids. Indeed, instead of the typical lipids commonly found in Bacteria and Eukarya, which are built upon straight fatty-acyl chains ester-linked to a glycerol backbone in a sn-1,2 configuration, archaeal lipids contain polyisoprenoid alkyl chains that are ether-linked to a glycerol in a sn-2,3 configuration (De Rosa and Gambacorta, 1988). While polyisoprenoid alkyl chains provide greater membrane packing and impermeability compared to fatty acyl chains due to their heavy branching (Komatsu and Chong, 1998), ether bonds are more chemically and thermally resistant than ester linkages and less polar (Baba et al., 1999), both contributing to the enhanced stability of archaeal membranes. In addition, most Archaea are also capable of synthesizing both bilayer-forming diether lipids (one polar moiety) and membrane-spanning tetraether lipids (two polar moieties), the latter generating membrane monolayers that are even more rigid and impermeable than archaeal bilayers (Chong, 2010). In contrast to their core lipids, the polar head groups of archaeal lipids remain very similar to those of Bacteria and Eukarya, with phospho- and glyco-lipids deriving from sugars (glycerol, inositol, glucose, and $\mathrm{N}$-acetylhexosamine) (Jensen et al., 2015), aminoacids (serine and ethanolamine) (Koga et al., 1993), or combinations of both (Koga et al., 1993). Although substantial lipidomes, e.g., 
Please cite this article as: Tourte, M. et al. (2021). Acid Hydrolysis for the Extraction of Archaeal Core Lipids and HPLC-MS Analysis. Bio-protocol 11(16): e4118. DOI: $10.21769 /$ BioProtoc. 4118 .

containing up to 100 structures, have been described for Archaea (e.g., Elling et al., 2017; Bale et al., 2019), those remain less diverse than the lipidomes of Bacteria and Eukarya, which comprise hundreds to thousands of different lipid structures (Gerl et al., 2012). In addition to this large difference between archaeal and other typical lipidomes, several studies have reported biases in archaeal lipid extraction and detection (Huguet et al., 2010; Cario et al., 2015), suggesting that a significant part of the archaeal lipidome might remain inaccessible to current methodologies. The absence of procedures to completely extract and analyze archaeal lipids currently hinders our understanding of their membrane physiology and adaptation. However, partial comprehension of these phenomena can easily and reproducibly be obtained by the removal of the lipids' polar head groups, granting access to exhaustive, and thus precise, archaeal core lipid compositions (De Rosa et al., 1980; Trincone et al., 1992; Hopmans et al., 2000; Cario et al., 2015).

\section{Materials and Reagents}

1. Pyrex ${ }^{\mathrm{TM}}$ tubes with PTFE caps (Fisher Scientific, catalog number: 10004654)

2. Glass pipettes and hand pump (Fisher Scientific, catalog number: 11546963)

3. 1 ml-glass syringe (gastight Series 1000; Hamilton, catalog number: 81320)

4. PTFE glass vial caps (Agilent Technologies, catalog number: 5182-0719)

5. Glass bottles (Dutscher, catalog number: 090971)

6. 50-ml round bottomed flasks (Fisher Scientific, catalog number: CG150689)

7. Glass funnels (DWK Life Sciences, catalog number: 213514609)

8. 2 ml-glass vials (Sodipro, catalog number: 2108081)

9. Freeze-dried archaeal biomass

10. Sand (VWR, catalog number: 27460.364 )

11. Glass wool (Fisher Scientific, catalog number: 12373866)

12. Celite ${ }^{\mathrm{TM}}$ (ACROS Organics, catalog number: 349675000$)$

13. Silica gel Geduran Si $60^{\circledR}$ (Merck, catalog number 1.11567)

14. Hydrochloric acid 37\% (HCl; Fisher Scientific, catalog number: 10294190)

15. Deionized water $\left(\mathrm{H}_{2} \mathrm{O}\right)$

16. Methanol (MeOH; ACROS Organics, catalog number: 364390025$)$

17. Dichloromethane (DCM; ACROS Organics, catalog number: 326850025)

18. Acetone (ACROS Organics, catalog number: 444150050)

19. $n$-Heptane (Hept; ACROS Organics, catalog number: 364360025$)$

20. Isopropyl alcohol (or propan-2-ol; IPA; ACROS Organics, catalog number: 184130250)

\section{Equipment}

1. Oven (up to $450^{\circ} \mathrm{C}$ )

2. Vacuum pump (Edwards Vacuum, catalog number: A34317984) 
Please cite this article as: Tourte, M. et al. (2021). Acid Hydrolysis for the Extraction of Archaeal Core Lipids and HPLC-MS Analysis. Bio-protocol 11(16): e4118. DOI: 10.21769/BioProtoc. 4118 .

Note: Here, we used a vacuum pump at $50 \mathrm{~Hz}$ with a maximum displacement of $20.5 \mathrm{~m}^{3} \mathrm{~h}^{-1}$, a maximum pumping speed of $17.0 \mathrm{~m}^{3} \mathrm{~h}^{-1}$, and an ultimate vacuum of $2 \times 10^{-2} \mathrm{mbar}$. Although a strong suction power allows for a faster procedure, any vacuum pump able to reach an ultimate vacuum below 20 mbar can be used.

3. Rotary evaporator (Büchi, models: Rotavapor RE 111; heating bath, B-491)

Note: Any rotary evaporator compatible with your vacuum pump and heating bath up to $50^{\circ} \mathrm{C}$ can be used.

4. $\mathrm{N}_{2}$ drying system

Note: Here, we connected our building $N_{2}$ supply (Air Products, CryoEase ${ }^{\circledR}$ supply system) to a distributing system consisting of polycarbonate tubes (Cole-Parmer, catalog number: FV30526-18) and splitting valves (Cole-Parmer, catalog number: FV-30600-02) ending on needles (BD, catalog number: 300700) or glass pipettes. Refer to Figure 3 for a depiction of the system.

5. High performance liquid chromatography (HPLC) instrument

Note: Here, we used a HP 1100 series LC system equipped with binary pumps (Agilent G1312A), a solvent degasser (Agilent G1379A), an autoinjector (Agilent G1913A), a thermostated column compartment (Agilent G1316A), and Chemstation chromatography manager software (Agilent Technologies Rev. A.09.03). However, any HPLC system that can function with a solvent gradient can be used.

6. Prevail Cyano 3 microns column $(150 \mathrm{~mm} \times 2.1 \mathrm{~mm}$; Grace Davison Discovery Sciences, VWR, catalog number: $\mathrm{HICH} 99243)$

Note: When not used, our column was stored in Hept/IPA (95:5, v/v). However, the solvent mixture allows for both the storage and equilibration of the column and thus depends on the separation gradient (see Step D4). It might hence be adjusted to your requirements.

7. Mass spectrometer equipped with an ion trap and an atmospheric pressure chemical ionization (APCl) source

Note: Here, we used an Ion Trap MS Esquire 3000Plus (Bruker Daltonics, GE0100-G552) and an Agilent APCl source (Agilent G1947A), but any Mass spectrometry (MS) instrument equipped with an $\mathrm{APCl}$ source can be used.

\section{Software}

Mass spectrum and chromatogram processing software.

Note: Here, we used the Compass Data Analysis software (version 5.0, 2017) provided by Bruker Daltonics, but any mass spectrum and chromatogram processing software, especially the one provided by your HPLC-MS instrument manufacturer, can be used. 
Please cite this article as: Tourte, M. et al. (2021). Acid Hydrolysis for the Extraction of Archaeal Core Lipids and HPLC-MS Analysis. Bio-protocol 11(16): e4118. DOI: $10.21769 /$ BioProtoc. 4118 .

\section{Procedure}

\section{Material preparation}

1. Archaeal biomass must be freeze-dried beforehand.

Note: As for every lipid extraction procedure, the growth medium may contain a significant amount of lipids unrelated to the cells to be analyzed. To avoid extracting these lipids from the culture medium, it is important to rinse the cells twice with an appropriate isotonic solution prior to freezedrying.

2. Remove all traces of potential organic contaminants by combusting all glassware, sand, celite, and glass wool at $450^{\circ} \mathrm{C}$ for $4 \mathrm{~h}$ before use. Rinse the glassware with deionized water, acetone, and DCM (in that order). Let it dry before use.

3. Silica gel is pre-extracted with DCM in a Soxhlet apparatus.

4. All reagents and solvents (except Hept) must be high grade, i.e., $>99 \%$ purity, to reduce the risk of contamination.

Note: High purity solvent can also be obtained by distillation if possible.

5. Hept is cleaned from organic contaminants by filtration over a column filled with silica gel.

6. Prepare $100 \mathrm{ml}$ of $1.2 \mathrm{~N} \mathrm{HCl}$ in $\mathrm{MeOH}$ by adding $10 \mathrm{ml}$ of $\mathrm{HCl} 37 \%$ to $90 \mathrm{ml}$ of $\mathrm{MeOH}$. Note: The solution can be stored for several weeks in a capped glass bottle at room temperature.

7. Prepare $100 \mathrm{ml}$ of $\mathrm{MeOH} / \mathrm{DCM}(1: 1, \mathrm{v} / \mathrm{v})$ by adding $50 \mathrm{ml}$ of $\mathrm{DCM}$ into $50 \mathrm{ml}$ of $\mathrm{MeOH}$. Note: The solution can be stored in a capped glass bottle at room temperature for several weeks.

8. Prepare $50 \mathrm{ml}$ of Hept/IPA $(99: 1, \mathrm{v} / \mathrm{v})$ by adding $0.5 \mathrm{ml}$ of IPA to $49.5 \mathrm{ml}$ of Hept. Note: The solution can be stored for several weeks in a capped glass flask at room temperature.

9. Prepare $1,000 \mathrm{ml}$ of Hept/IPA (95:5, v/v) by adding $50 \mathrm{ml}$ of IPA to 950 of Hept. Note: The solution can be stored for several weeks in a glass bottle at room temperature.

10. Prepare a 1,000-ml glass bottle filled with Hept.

Note: The solution can be stored for several weeks at room temperature.

A. Archaeal cell hydrolysis (Figure 1)

1. Transfer the archaeal cell pellet to a Pyrex ${ }^{\mathrm{TM}}$ glass tube using a small glass funnel.

2. Use $4 \mathrm{ml}$ of $1.2 \mathrm{~N} \mathrm{HCl}$ in $\mathrm{MeOH}$ to resuspend the archaeal cell pellet and rinse the glass funnel using glass pipettes and a hand pump.

Note: The volume at this step may be modified depending on the size of the cell pellet. However, do not fill the Pyrex ${ }^{T M}$ tube above half of its total volume.

3. Tightly close the Pyrex ${ }^{\mathrm{TM}}$ tube with a cap containing a PTFE seal.

Note: Double check that the cap is closed to avoid any evaporation that could lead to the destruction of the sample.

4. To avoid any cross-contamination of samples and contamination of the oven in case of leakage, put $5 \mathrm{~cm}$ of sand in a glass bottle, place your Pyrex ${ }^{\mathrm{TM}}$ tubes in the sand, and close the bottle with a stopper. 
Please cite this article as: Tourte, M. et al. (2021). Acid Hydrolysis for the Extraction of Archaeal Core Lipids and HPLC-MS Analysis. Bio-protocol 11(16): e4118. DOI: 10.21769/BioProtoc. 4118 .

5. Heat your samples at $110^{\circ} \mathrm{C}$ for $4 \mathrm{~h}$.

Note: This procedure is strong enough to remove most of the archaeal polar head groups without further degrading archaeal lipids, i.e., ether linkages remain unharmed.

6. Take your samples out of the oven and let them cool down to room temperature.

Note: At this step, if your tubes were not sealed tightly enough, your sample might have dried out and completely burned (black-brown color of the sample), which might result in the destruction of core lipids. As a quality control of the procedure, check that tubes still contain solvent and that cell pellets are almost completely disrupted and colorless.

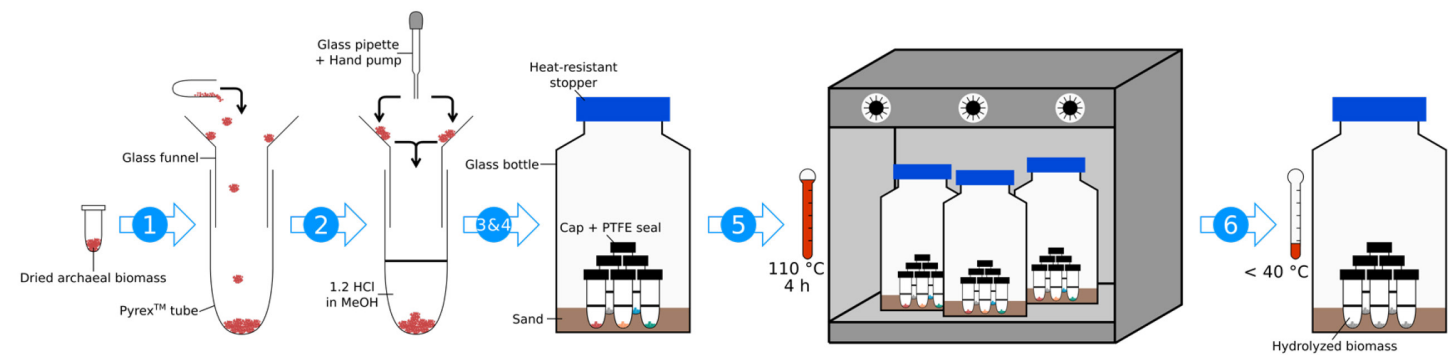

Figure 1. Schematic of the workflow for the direct acid methanolysis of archaeal dried biomass. Circled numbers refer to the steps in the text. Abbreviations: $\mathrm{MeOH}$, methanol; PTFE, polytetrafluoroethylene.

B. Core lipid extraction (Figure 2)

1. Once your sample has cooled down, carefully open the Pyrex ${ }^{\mathrm{TM}}$ tube.

Warning: Due to the gas generated during the heating step, hot gas and liquid may gush out from the tube when opening it.

2. Add $4 \mathrm{ml}$ of DCM into the Pyrex ${ }^{\mathrm{TM}}$ tube (final MeOH/DCM 1:1, v/v). Note: The volume at this step may be modified depending on the size of the cell pellet.

3. Create a filter by placing glass wool and then celite into a glass funnel. Place the glass funnel onto a $50 \mathrm{ml}$-round-bottomed flask suited for the rotary evaporator.

4. Transfer the sample onto the filter and filter it through the funnel into the flask to remove the solid particles (i.e., cell debris) from the lipid extract.

5. Rinse the Pyrex ${ }^{\mathrm{TM}}$ tube twice with $4 \mathrm{ml}$ of $\mathrm{MeOH} / \mathrm{DCM}(1: 1, \mathrm{v} / \mathrm{v})$ and filter it into the same flask as above.

6. Rinse the filter into the same flask twice with $4 \mathrm{ml}$ of $\mathrm{MeOH} / \mathrm{DCM}(1: 1, \mathrm{v} / \mathrm{v})$. 


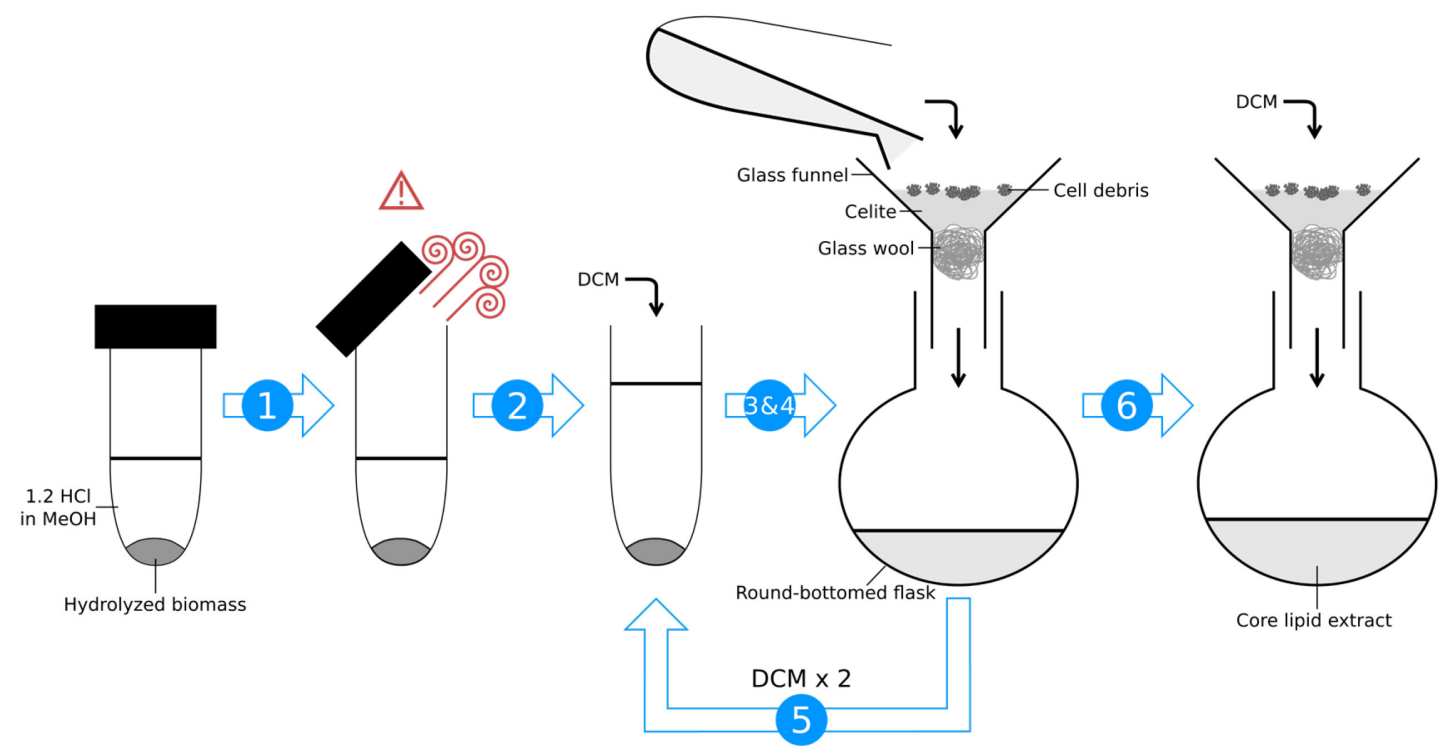

Figure 2. Schematic of the workflow for the extraction of core lipids from the hydrolyzed biomass. Circled numbers refer to the steps in the text. Abbreviations: $\mathrm{MeOH}$, methanol; DCM, dichloromethane.

C. Sample preparation for HPLC analysis (Figure 3)

1. Dry the sample under reduced pressure using a rotary evaporator set to $40^{\circ} \mathrm{C}$.

2. Rinse the flask with $\mathrm{MeOH}$ and dry again under reduced pressure to allow for the complete evaporation of the remaining $\mathrm{HCl} / \mathrm{H}_{2} \mathrm{O}$.

3. Once dried, carefully dissolve the content of the flask with $0.5 \mathrm{ml}$ of Hept/IPA $(99: 1, \mathrm{v} / \mathrm{v})$ to recover core lipids and transfer into a $2 \mathrm{ml}$-vial using a glass syringe.

4. Rinse the flask three more times and transfer into the $2 \mathrm{ml}$-vial.

5. Dry the sample under a steam of $\mathrm{N}_{2}$.

6. Resuspend the sample into $1 \mathrm{ml}$ of Hept/IPA $(99: 1, \mathrm{v} / \mathrm{v})$ prior to HPLC analysis.

Note: The lipid extract can be stored at $-20^{\circ} \mathrm{C}$ for long periods of time (i.e., several years).

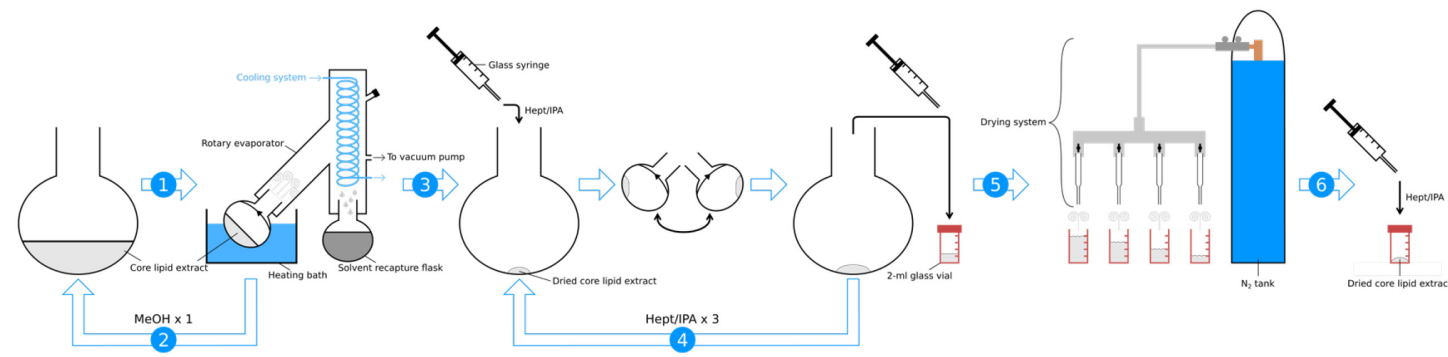

Figure 3. Schematic of the workflow for the core lipid extract preparation for HPLC analysis. Circled numbers refer to the steps in the text. Abbreviations: $\mathrm{MeOH}$, methanol; Hept, heptane; IPA, isopropanol.

D. HPLC-MS core lipid separation and analysis 
Please cite this article as: Tourte, M. et al. (2021). Acid Hydrolysis for the Extraction of Archaeal Core Lipids and HPLC-MS Analysis. Bio-protocol 11(16): e4118. DOI: $10.21769 /$ BioProtoc. 4118 .

Note: This section is based on Tourte et al. (2020), but the nature and volume of solvents can be adjusted to fit the requirements of your chromatographic separation, i.e., nature and size of the column.

1. Start your chromatography instrument.

2. Set the temperature of the column thermostat to $30^{\circ} \mathrm{C}$.

3. Empty the trash collectors and fill the vial used for rinsing (Hept//PA 95:5, v/v).

4. Feed the chromatography manager software with the gradient used for HPLC chromatography. Note: Here, we used the following gradient with $A=\operatorname{HeptIPA}(95: 5, v / v)$ and $B=$ Hept, and a flow rate of $0.2 \mathrm{ml} \mathrm{min}^{-1}: 95 \% \mathrm{~B}$ (5 min isocratic) to $65 \% \mathrm{~B}$ in $30 \mathrm{~min}$ ( 5 min isocratic), then to $0 \% \mathrm{~B}$ in $1 \mathrm{~min}$ (10 min isocratic), and back to $95 \%$ B in $1 \mathrm{~min}$ ( $5 \mathrm{~min}$ isocratic). The total run time per sample is $57 \mathrm{~min}$.

5. Double-check for stable pressure in the system to ensure proper separation. Flush your system if necessary.

6. Start the MS instrument.

7. Feed the MS manager software with the conditions for the MS analysis.

Note: Here, we used the following MS conditions: nebulizer pressure, $50 \mathrm{psi}$; APCI temperature, $420^{\circ} \mathrm{C}$; drying temperature, $350^{\circ} \mathrm{C}$; drying gas $\left(\mathrm{N}_{2}\right)$ flow, $5 \mathrm{~L} \mathrm{~min}^{-1}$; capillary voltage, $\quad-2 \quad k V$; corona, $4 \mu \mathrm{A}$; and scan range $\mathrm{m} / \mathrm{z}, 600-2,200$.

8. Double-check that all parameters properly reach the set values.

9. Place the $2 \mathrm{ml}$-vials containing your samples dissolved in $1 \mathrm{ml}$ of Hept/IPA (99:1, v/v; see Step C6) onto the HPLC autosampler.

10. Fill the chromatography manager software file with your sample information and sequence. Note: The injection volume is set to $10 \mu$. To enhance reproducibility, adjust the concentration of your samples (Step C6) rather than change the injection volume.

11. Run your sequence.

Note: Run a control sample every 30-40 analyses to check for appropriate separation and intensity. Reproducibility is evaluated by running selected samples in duplicate or triplicate and a reference sample. For instance, as a reference sample, we used a core lipid extract from Pyrococcus furiosus, which contains diphytanyl glycerol diethers (DGD) and multiple isomers of tetraether lipids, i.e., glycerol mono-, di-, and trialkyl glycerol tetraethers (GMGT, GDGT, and GTGT, respectively) with 0 to 4 cyclopentane rings, to assess the performance of the HPLC column. Additionally, run a standard mixture (synthetic archaeal-like lipids can be purchased from Avanti) to assess the relative response factors of the compounds detected. As a standard mixture, we used a solution of DGD/GDGTO at 2:1 molar ratio which gave a relative response factor of $D G D$ ca. 10 times lower than that of GDGTO under our analytical conditions (Tourte et al., 2020).

E. Chromatogram and mass spectrum analysis

Note: Relatively few examples of archaeal core lipid mass spectra have been published, but 
Please cite this article as: Tourte, M. et al. (2021). Acid Hydrolysis for the Extraction of Archaeal Core Lipids and HPLC-MS Analysis. Bio-protocol 11(16): e4118. DOI: 10.21769/BioProtoc.4118.

identification can be aided by referring to, e.g., Hopmans et al. (2000).

1. Draw the total ion current (TIC) of your analysis using mass spectrum and chromatogram processing software.

2. Major compounds, i.e., the most abundant on the TIC, are tentatively identified based on their mass spectra, fragmentation patterns, retention time in LC, and comparison with published data (where possible). Use an average of several spectra of a given core lipid peak to ensure proper identification (Figure 1).

Note: Very few ions besides the protonated adduct of the intact compound are generated by our APCI procedure, and compounds are thus identified based on their protonated masses. To further ascertain the structures of identified core lipids, MS/MS can be performed.

3. Draw the extracted ion chromatogram (EIC) by extracting the masses of all compounds identified (Figure 4). For each compound, select a mass range suited to your MS system. Note: Considering our system, we usually use a mass range of \pm 0.5 .

4. Integrate each peak on your EIC to determine the relative proportion of each core lipid and correct with the response factor of the compounds under your analytical conditions (see Step D11).

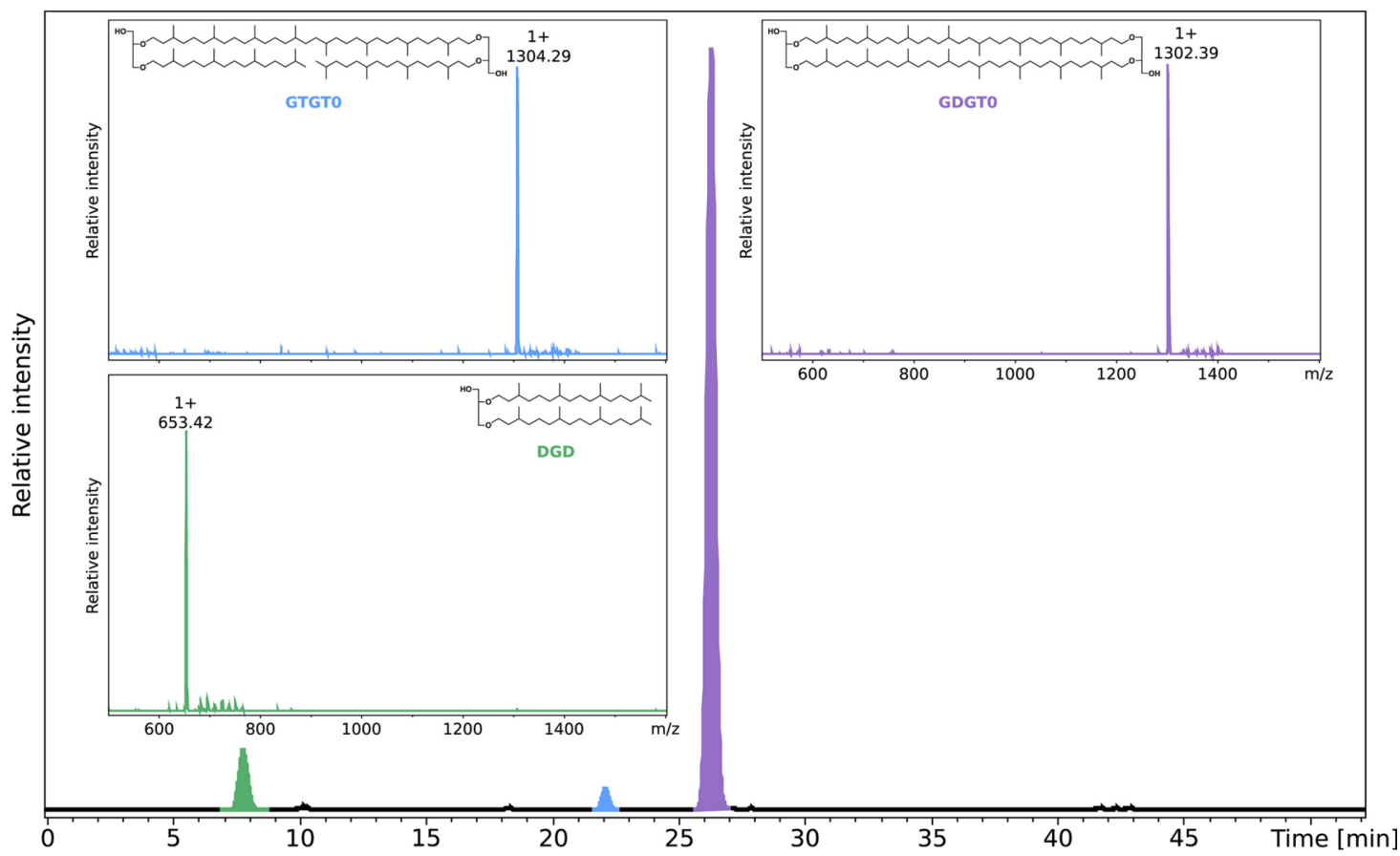

Figure 4. Core lipid analysis of Thermococcus acidaminovorans. Cells were grown under optimal conditions $\left(85^{\circ} \mathrm{C}, \mathrm{pH} 9.0,3.0 \% \mathrm{NaCl}(\mathrm{w} / \mathrm{v})\right.$; Dirmeier et al., 1998). The chromatogram was drawn by extracting the following masses: $653.5,1302.5$, and 1304.5. Inserts show the mass spectrum and the resulting core structure for each colored peak: green, diphytanyl glycerol diether (DGD); blue, glycerol trialkyl glycerol tetraether with no cyclopentane ring (GTGT0); and purple, glycerol dibiphytanyl glycerol tetraether with no cyclopentane ring (GDGT0). The indicated masses correspond to protonated adducts. Correcting for the response factors 
Please cite this article as: Tourte, M. et al. (2021). Acid Hydrolysis for the Extraction of Archaeal Core Lipids and HPLC-MS Analysis. Bio-protocol 11(16): e4118. DOI: 10.21769/BioProtoc.4118.

observed under our analytical conditions, DGD, GTGT0, and GDGT0 had relative proportions of 46,1 , and $53 \%$, respectively.

\section{Acknowledgments}

The authors would like to thank the French National Research Agency for funding the ArchaeoMembranes project (ANR-17-CE11-0012-01) and the CNRS interdisciplinary program 'Origines' for funding the ReseArch project.

\section{Competing interests}

The authors declare no conflicts of interest.

\section{$\underline{\text { References }}$}

1. Baba, T., Toshima, Y., Minamikawa, H., Hato, M., Suzuki, K. and Kamo, N. (1999). Formation and characterization of planar lipid bilayer membranes from synthetic phytanyl-chained glycolipids. Biochim Biophys Acta-Biomembr 1421: 91-102.

2. Bale, N. J., Sorokin, D. Y., Hopmans, E. C., Koenen, M., Rijpstra, W. I. C., Villanueva, L., Wienk, H. and Damsté, J. S. S. (2019). New insights into the polar lipid composition of extremely halo (alkali) philic euryarchaea from hypersaline lakes. Front Microbiol 10: 377.

3. De Rosa, M., Gambacorta, A., Nicolaus, B. and Bu'Lock, J. D. (1980). Complex lipids of Caldariella acidophila, a thermoacidophile archaebacterium. Phytochemistry 19: 821-825.

4. Trincone, A., Nicolaus, B., Palmieri, G., De Rosa, M., Huber, R., Huber, G., Stetter, K.O. and Gambacorta, A. (1992). Distribution of complex and core lipids within new hyperthermophilic members of the Archaea domain. Syst Appl Microbiol 15: 11-17.

5. Hopmans, E. C., Schouten, S., Pancost, R. D., van der Meer, M. T. and Sinninghe Damste, J. S. (2000). Analysis of intact tetraether lipids in archaeal cell material and sediments by high performance liquid chromatography/atmospheric pressure chemical ionization mass spectrometry. Rapid Commun Mass Spectrom 14(7): 585-589.

6. Cario, A., Grossi, V., Schaeffer, P. and Oger, P. M. (2015). Membrane homeoviscous adaptation in the piezo-hyperthermophilic archaeon Thermococcus barophilus. Front Microbiol 6:1152.

7. Chong, P. L.-G. (2010). Archaebacterial bipolar tetraether lipids: physico-chemical and membrane properties. Chem Phys Lipids 163: 253-265.

8. De Rosa, M. and Gambacorta, A. (1988). The lipids of archaebacteria. Prog Lipid Res 27: 153175.

9. Dirmeier, R., Keller, M., Hafenbradl D., Braun, F-J., Rachel, R., Burggraf, S. and Stetter K. O. (1998). Thermococcus acidaminovorans sp. nov., a new hyperthermophilic alkalophilic archaeon growing on amino acids. Extremophiles 2: 109-114. 
Please cite this article as: Tourte, M. et al. (2021). Acid Hydrolysis for the Extraction of Archaeal Core Lipids and HPLC-MS Analysis. Bio-protocol 11(16): e4118. DOI: 10.21769/BioProtoc. 4118 .

10. Elling, F. J., Könneke, M., Nicol, G. W., Stieglmeier, M., Bayer, B., Spieck, E., de la Torre, J. R., Becker, K. W., Thomm, M., Prosser, J. I., Herndl, G. J., Schleper, C. and Hinrichs, K.-U. (2017). Chemotaxonomic characterisation of the thaumarchaeal lipidome. Environ Microbiol 19: 26812700.

11. Gerl, M. J., Sampaio, J. L., Urban, S., Kalvodova, L., Verbavatz, J.-M., Binnington, B., Lindemann, D., Lingwood, C. A., Shevchenko, A., Schroeder, C. and Simons, K. (2012). Quantitative analysis of the lipidomes of the influenza virus envelope and MDCK cell apical membrane. J Cell Biol 196: 213-221.

12. Huguet, C., Martens-Habbena, W., Urakawa, H., Stahl, D. A. and Ingalls, A. E. (2010). Comparison of extraction methods for quantitative analysis of core and intact polar glycerol dialkyl glycerol tetraethers (GDGTs) in environmental samples. Limnol Oceanogr Meth 8: 127145.

13. Jensen, S. M., Brandl, M., Treusch, A. H. and Ejsing, C. S. (2015). Structural characterization of ether lipids from the archaeon Sulfolobus islandicus by high-resolution shotgun lipidomics. $J$ Mass Spectrom 50: 476-487.

14. Koga, Y., Akagawa-Matsushita, M., Ohga, M. and Nishihara, M. (1993). Taxonomic significance of the distribution of component parts of polar ether lipids in methanogens. Syst Appl Microbiol 16: 342-351.

15. Komatsu, H. and Chong, P. L.-G. (1998). Low permeability of liposomal membranes composed of bipolar tetraether lipids from thermoacidophilic archaebacterium Sulfolobus acidocaldarius. Biochemistry 37: 107-115.

16. Tourte, M., Schaeffer, P., Grossi, V. and Oger, P. M. (2020). Functionalized membrane domains: an ancestral feature of Archaea? Front Microbiol 11: 526. 